FACTA UNIVERSITATIS (NIŠ)

Ser. Math. Inform. Vol. 35, No 1 (2020), 243-252

https://doi.org/10.22190/FUMI2001243C

\title{
THE ANALYTIC SOLUTION OF INITIAL BOUNDARY VALUE PROBLEM INCLUDING TIME FRACTIONAL DIFFUSION EQUATION
}

\author{
Süleyman Çetinkaya, Ali Demir and Hülya Kodal Sevindir
}

C 2020 by University of Niš, Serbia | Creative Commons License: CC BY-NC-ND

\begin{abstract}
The motivation of this study is to determine the analytic solution of initial boundary value problem including time fractional differential equation with Neumann boundary conditions in one dimension. By making use of seperation of variables, the solution is constructed in the form of a Fourier series with respect to the eigenfunctions of a corresponding Sturm-Liouville eigenvalue problem.

Keywords: Caputo fractional derivative, space-fractional diffusion equation, MittagLeffler function, initial-boundary-value problems, spectral method.
\end{abstract}

\section{Introduction}

As PDEs of fractional order play an important role in modelling numerous processes and systems in various scientific research areas such as applied mathematics, physics chemistry etc., the interest in this topic has become enourmous. Since the fractional derivative is non-local, the model with fractional derivative for physical problems turns out to be the best choice to analyze the behaviour of the complex non linear processes. That is why this has attracted an increasing number of researchers. The derivatives in the sense of Caputo is one of the most common since modelling of physical processes with fractional differential equations including Caputo derivative is much better than other models. In literature, increasing number of studies can be found supporting this conclusion [1], [2], [3], [4], [5], [6], [7], [8], [9], [10], [11], [12], [14], [15], [16], [17]. Especially there are various studies on fractional diffusion equations: Exact analytical solutions of heat equations are obtained by using operational method [18]. The existence, uniqueness and regularity of solution of impulsive sub-diffusion equation are established by means of eigenfunction expansion [19]. The anomalous diffusion models with non-singular power-law kernel have been investigated and constructed [20]. Moreover, the Caputo derivative of constant is zero which is not hold by many fractional derivatives. The solutions of fractional PDEs and ODEs are determined in terms of Mittag-Leffler function.

Received September 12, 2019; accepted February 20, 2020

2010 Mathematics Subject Classification. Primary 26A33; Secondary 65M70 


\section{Preliminary Results}

In this section, we recall fundamental definition and well known results about fractional derivative in Caputo sense.

Definition 2.1. The $q^{t h}$ order fractional derivative of $u(t)$ in Caputo sense is defined as

$$
D^{q} u(t)=\frac{1}{\Gamma(n-q)} \int_{t_{0}}^{t}(t-s)^{n-q-1} u^{(n)}(s) d s, t \in\left[t_{0}, t_{0}+T\right]
$$

where $u^{(n)}(t)=\frac{d^{n} u}{d t^{n}}, n-1<q<n$. Note that Caputo fractional derivative is equal to integer order derivative when the order of the derivative is integer.

Definition 2.2. The $q^{t h}$ order Caputo fractional derivative for $0<q<1$ is defined as

$$
D^{q} u(t)=\frac{1}{\Gamma(1-q)} \int_{t_{0}}^{t}(t-s)^{-q} u^{\prime}(s) d s, t \in\left[t_{0}, t_{0}+T\right]
$$

The two-parameter Mittag-Leffler function which is taken into account in eigenvalue problem, is given by

$$
E_{\alpha, \beta}\left(\lambda\left(t-t_{0}\right)^{\alpha}\right)=\sum_{k=0}^{\infty} \frac{\left(\lambda\left(t-t_{0}\right)^{\alpha}\right)^{k}}{\Gamma(\alpha k+\beta)}, \alpha, \beta>0
$$

including constant $\lambda$. Especially, for $t_{0}=0, \alpha=\beta=q$ we have

$$
E_{\alpha, \beta}\left(\lambda t^{q}\right)=\sum_{k=0}^{\infty} \frac{\left(\lambda t^{q}\right)^{k}}{\Gamma(q k+q)}, q>0
$$

Mittag-Leffler function coincides with exponential function i.e., $E_{1,1}(\lambda t)=e^{\lambda t}$ for $q=1$. For details see $[13,21]$.

We determined the solution of following time fractional differential equation with Neumann boundary and initial conditions in this study:

$$
\begin{gathered}
D_{t}^{\alpha} u(x, t ; \alpha)=u_{x x}(x, t ; \alpha)-\gamma u(x, t ; \alpha), \\
u_{x}(0, t)=u_{x}(l, t)=0, \\
u(x, 0)=f(x)
\end{gathered}
$$

where $0<\alpha<1,0 \leqslant x \leqslant l, 0 \leqslant t \leqslant T, \gamma \in \mathbb{R}$. 


\section{Main Results}

By means of separation of variables method, the solution to the problem (2.5)(2.7) is constructed in analytical form. Thus, a solution to the problem (2.5)-(2.7) has the following form:

$$
u(x, t ; \alpha)=X(x) T(t ; \alpha)
$$

where $0 \leqslant x \leqslant l, 0 \leqslant t \leqslant T$.

Plugging (3.1) into (2.5) and arranging it, we have

$$
\frac{D_{t}^{\alpha}(T(t ; \alpha))}{T(t ; \alpha)}+\gamma=\frac{X^{\prime \prime}(x)}{X(x)}=-\lambda^{2}
$$

The equation (3.2) produces a fractional differential equation with respect to time and an ordinary differential equation with respect to space. The first ordinary differential equation is obtained by taking the equation on the right hand side of Eq. (3.2). Hence, with boundary conditions (2.6), we have the following problem:

$$
\begin{gathered}
X^{\prime \prime}(x)+\lambda^{2} X(x)=0 \\
X^{\prime}(0)=X^{\prime}(l)=0
\end{gathered}
$$

The solution of eigenvalue problem (3.3)-(3.4) is accomplished by making use of the exponantial function of the following form:

$$
X(x)=e^{r x}
$$

Hence, the characteristic equation is computed in the following form:

$$
r^{2}+\lambda^{2}=0
$$

Case 1. If $\lambda=0$, the Eq.(3.6) has two coincident roots $r_{1}=r_{2}$, leading to the general solution of the eigenvalue problem (3.3)-(3.4) having the following form:

$$
\begin{gathered}
X(x)=k_{1} x+k_{2} \\
X^{\prime}(x)=k_{1}
\end{gathered}
$$

The first boundary condition yields

$$
X^{\prime}(0)=k_{1}=0 \Rightarrow k_{1}=0
$$

This result leads to 


$$
X(x)=k_{2}
$$

Similarly, the second boundary condition leads to

$$
X^{\prime}(l)=k_{1}=0 \Rightarrow k_{1}=0
$$

Hence, we obtain the solution as follows:

$$
X_{0}(x)=k_{2}
$$

Case 2. If $\lambda<0$, the Eq.(3.6) has distinct real roots $r_{1}, r_{2}$ leading to the general solution of the eigenvalue problem (3.3)-(3.4) and having the following form:

$$
\begin{gathered}
X(x)=c_{1} e^{r_{1} x}+c_{2} e^{r_{2} x} \\
X^{\prime}(x)=r_{1} c_{1} e^{r_{1} x}+r_{2} c_{2} e^{r_{2} x}
\end{gathered}
$$

The first boundary condition yields

$$
X^{\prime}(0)=r_{1} c_{1}+r_{2} c_{2}=0 \Rightarrow c_{1}=-\frac{r_{2}}{r_{1}} c_{2}
$$

This result leads to

$$
X(x)=-\frac{r_{2}}{r_{1}} c_{2} e^{r_{1} x}+c_{2} e^{r_{2} x}
$$

Similarly, the last boundary condition leads to

$$
X(l)=-\frac{r_{2}}{r_{1}} c_{2} e^{r_{1} l}+c_{2} e^{r_{2} l}=0 \Rightarrow c_{2}=0
$$

which implies that $c_{1}=0$. Therefore, $X(x)=0$ which means that we don't have any solution for $\lambda<0$.

Case 3. If $\lambda>0$, the Eq.(3.6) has two complex conjugate roots lead to the general solution of the eigenvalue problem (3.3)-(3.4) and have the following form:

$$
\begin{gathered}
X(x)=c_{1} \cos (\lambda x)+c_{2} \sin (\lambda x) \\
X^{\prime}(x)=-c_{1} \lambda \sin (\lambda x)+c_{2} \lambda \cos (\lambda x)
\end{gathered}
$$

The first boundary condition yields

$$
X^{\prime}(0)=0=c_{2} \lambda \Rightarrow c_{2}=0
$$


This result leads to

$$
X(x)=c_{1} \cos (\lambda x)
$$

Similarly, the last boundary condition leads to

$$
X^{\prime}(l)=-c_{1} \lambda \sin (\lambda l)=0
$$

which implies that

$$
\sin (\lambda l)=0
$$

Let $n \pi=\lambda_{n} l$. Hence, the eigenvalues can be determined as follows:

$$
\lambda_{n}=\frac{n \pi}{l}, \lambda_{1}<\lambda_{2}<\lambda_{3}<\ldots
$$

The representation of the solution is obtained as follows:

$$
X_{n}(x)=\cos \left(\frac{n \pi x}{l}\right), n=1,2,3, \ldots
$$

The second equation in (3.2) for every eigenvalue $\lambda_{n}$ is determined as follows:

$$
\frac{D_{t}^{\alpha}(T(t ; \alpha))}{T(t ; \alpha)}=-\left(\lambda^{2}+\gamma\right)
$$

which yields the following solution

$$
T_{n}(t ; \alpha)=E_{\alpha, 1}\left(-\left(\left(\frac{n \pi x}{l}\right)^{2}+\gamma\right) t^{\alpha}\right) n=1,2,3, \ldots
$$

The solution for every eigenvalue $\lambda_{n}$ is constructed as follows:

$u_{n}(x, t ; \alpha)=X_{n}(x) T_{n}(t ; \alpha)=E_{\alpha, 1}\left(-\left(\left(\frac{n \pi x}{l}\right)^{2}+\gamma\right) t^{\alpha}\right) \cos \left(\frac{n \pi x}{l}\right), n=0,1,2,3, \ldots$

Hence the general solution becomes

$$
u(x, t ; \alpha)=d_{0}+\sum_{n=1}^{\infty} d_{n} \cos \left(\frac{n \pi x}{l}\right) E_{\alpha, 1}\left(-\left(\left(\frac{n \pi x}{l}\right)^{2}+\gamma\right) t^{\alpha}\right)
$$

Note that boundary conditions and fractional differential equation are satisfied by this solution. The coefficients in (3.29) are obtained by making use of initial condition (2.7): 


$$
u(x, 0)=f(x)=d_{0}+\sum_{n=1}^{\infty} d_{n} \cos \left(\frac{n \pi x}{l}\right)
$$

$\left\langle f(x), \cos \left(\frac{k \pi x}{l}\right)\right\rangle=\left\langle d_{o}, \cos \left(\frac{k \pi x}{l}\right)\right\rangle+\sum_{n=1}^{\infty} d_{n}\left\langle\cos \left(\frac{n \pi x}{l}\right), \cos \left(\frac{k \pi x}{l}\right)\right\rangle$

We obtain the coefficients $d_{n}$ for $n=0,1,2,3, \ldots$ as follows:

$$
\begin{gathered}
d_{0}=\frac{1}{l} \int_{0}^{l} f(x) d x \\
d_{n}=\frac{2}{l} \int_{0}^{l} \cos \left(\frac{n \pi x}{l}\right) f(x) d x
\end{gathered}
$$

\section{Illustrative Example}

In this part, we first take the following partial differential equation with Neumann boundary and initial conditions:

$$
\begin{gathered}
u_{t}(x, t)=u_{x x}(x, t)-u(x, t), 0 \leqslant x \leqslant 1,0 \leqslant t \leqslant T \\
u_{x}(0, t)=0, u_{x}(1, t)=0,0 \leqslant t \leqslant T \\
u(x, 0)=\cos (\pi x) 0 \leqslant x \leqslant 1
\end{gathered}
$$

which has the solution in the following form:

$$
u(x, t)=\cos (\pi x) e^{-\left(\pi^{2}+1\right) t} .
$$

Secondly, we take the following time fractional differential equation with Neumann boundary and initial conditions:

$$
\begin{gathered}
D_{t}^{\alpha} u(x, t)=u_{x x}(x, t)-u(x, t), 0<\alpha<1,0 \leqslant x \leqslant 1,0 \leqslant t \leqslant T \\
u_{x}(0, t)=u_{x}(1, t)=0,0 \leqslant t \leqslant T \\
u(x, 0)=\cos (\pi x), 0 \leqslant x \leqslant 1
\end{gathered}
$$

The application of seperation of variables method yields the following equation: 


$$
\frac{D_{t}^{\alpha}(T(t ; \alpha))}{T(t ; \alpha)}+1=\frac{X^{\prime \prime}(x)}{X(x)}=-\lambda^{2}
$$

The equation (4.6) produces a fractional differential equation with respect to time and a differential equation with respect to space. The first fractional differential equation is obtained by taking the equation on the right hand side of Eq. (4.6). Hence, with boundary conditions (4.4), we have the following problem:

$$
\begin{aligned}
& X^{\prime \prime}(x)+\lambda^{2} X(x)=0 \\
& X^{\prime}(0)=0, X^{\prime}(1)=0
\end{aligned}
$$

Hence the eigenvalue problem (4.7)-(4.8) yields the following solution:

$$
X_{n}(x)=\cos (n \pi x), n=1,2,3, \ldots
$$

By using the similar calculations as in $(3.27), T_{n}(t ; \alpha)$ for $n=1,2,3, \ldots$ is determined in the following form:

$$
T_{n}(t ; \alpha)=E_{\alpha, 1}\left(-\left((n \pi)^{2}+1\right) t^{\alpha}\right) n=1,2,3, \ldots
$$

For each eigenvalue $\lambda_{n}$, we obtain the following solution:

$u_{n}(x, t ; \alpha)=X_{n}(x) T_{n}(t ; \alpha)=E_{\alpha, 1}\left(-\left((n \pi)^{2}+1\right) t^{\alpha}\right) \cos (n \pi x) n=0,1,2,3, \ldots$

Hence, the general solution is established as follows:

$$
u(x, t ; \alpha)=d_{0}+\sum_{n=1}^{\infty} d_{n} \cos (n \pi x) E_{\alpha, 1}\left(-\left((n \pi)^{2}+1\right) t^{\alpha}\right)
$$

Note that the general solution (4.12) satisfies both boundary conditions (4.4) and the fractional equation (4.3). We determine the coefficients $d_{n}$ in such a way that the general solution (4.12) satisfes the initial condition (4.5). Plugging $t=0$ in to the general solution (4.12) and making equal to the initial condition (4.5), we have

$$
u(x, 0)=d_{0}+\sum_{n=1}^{\infty} d_{n} \cos (n \pi x)
$$

Via the inner product we obtain the coefficients $d_{n}$ for $n=0,1,2,3, \ldots$ as follows:

$$
d_{0}=\frac{1}{l} \int_{0}^{1} f(x) d x=\int_{0}^{1} \cos (\pi x) d x=\left.\frac{1}{\pi} \sin (\pi x)\right|_{x=0} ^{x=1}=0
$$


and

$$
d_{n}=\frac{2}{l} \int_{0}^{1} \cos \left(\frac{n \pi x}{l}\right) f(x) d x=2 \int_{0}^{1} \cos (n \pi x) \cos (\pi x) d x
$$

Thus $d_{n}=0$ for $n \neq 1$.

For $n=1$ we get

$$
d_{1}=2 \int_{0}^{1} \cos ^{2}(\pi x) d x=\left.2\left[\frac{x}{2}+\frac{1}{4 \pi} \sin (2 \pi x)\right]\right|_{x=0} ^{x=1}=1
$$

Thus

$$
u(x, t ; \alpha)=\cos (\pi x) E_{\alpha, 1}\left(-\left(\pi^{2}+1\right) t^{\alpha}\right)
$$

It is important to note that plugging $\alpha=1$ in to the solution (4.17) gives the solution (4.2) which confirm the accuracy of the method we apply.

\section{Conclusion}

In this research, the analytic solution of initial boundary value problem with Neumann boundary conditions in one dimension has been constructed. By using the separation of variables, the solution is formed in the form of a Fourier series with respect to the eigenfunctions of a corresponding Sturm-Liouville eigenvalue problem.

\section{RE F E R E N C E S}

1. P. Agarwal, G. V. Milovanovic and S. K. Nisar: A Fractional Integral Operator Involving the Mittag-Leffler Type with Four Parameters. Facta Universitatis, Series: Mathematics and Informatics 305 (2015), 597-605.

2. M. A. BAYRAK and A. Demir: A new approach for space-time fractional partial dierential equations by Residual power series method. Appl. Math. And Comput. 336 (2013), 215-230.

3. M. A. BAYRAK and A. DemiR: Inverse Problem for Determination of An Unknown Coefficient in the Time Fractional Diffusion Equation. Communications in Mathematics and Applications 9 (2018), 229-237.

4. A. Benlabbes, M. Benbachir and M. Lakrib: Boundary value problems for nonlinear fractional differential equations. Facta Universitatis, Series: Mathematics and Informatics 302 (2015), 157-168.

5. A. Demir, M. A. Bayrak and E. Ozbilge: A new approach for the Approximate Analytical solution of space time fractional differential equations by the homotopy analysis method. Advances in mathematichal 2019 (2019), articleID 5602565 . 
6. A. Demir, S. Erman, B. ÖzGür and E. Korkmaz: Analysis of fractional partial differential equations by Taylor series expansion. Boundary Value Problems 201368 (2013).

7. A. DemiR, F. KANCA and E. ÖZBILGE: Numerical solution and distinguishability in time fractional parabolic equation. Boundary Value Problems 2015142 (2015).

8. A. Demir and E. ÖzBILGe: Analysis of the inverse problem in a time fractional parabolic equation with mixed boundary conditions. Boundary Value Problems 2014134 (2014).

9. S. ERman and A. Demir: A Novel Approach for the Stability Analysis of State Dependent Differential Equation. Communications in Mathematics and Applications 7 (2016), 105-113.

10. M. Houns and M. Bezziou: Existence and Stability Results for Fractional Differential Equations with Two Caputo Fractional Derivatives. Facta Universitatis, Series: Mathematics and Informatics 342 (2019), 341-357.

11. F. HuAng and F. LiU: The time-fractional diffusion equation and fractional advection-dispersion equation. The ANZIAM Journal 463 (2005), 317-330.

12. Y. LuCHKO: Initial boundary value problems for the one dimensional timefractional diffusion equation. Fractional Calculus and Applied Analysis 15 (2012), 141-160.

13. A. A. Kilbas, H. M. Srivastava and J. J. Trujillo: Theory and Applications of Fractional Differential Equations. Elsevier, Amsterdam, 2006.

14. Y. LuCHKo: Initial-boundary-value problems for the generalized multi-term timefractional diffusion equation. Journal of Mathematical Analysis and Applications 742 (2011), 538-548.

15. S. Momani and Z. ODIBAT: Numerical comparison of methods for solving linear differential equations of fractional order. Chaos Solitons and Fractals 315 (2007), $1248-1255$.

16. B. Özgür and A. Demir: Some Stability Charts of A Neural Field Model of Two Neural Populations. Communications in Mathematics and Applications 7 (2016), $159-166$.

17. L. Plociniczak: Analytical studies of a time-fractional porous medium equation. Derivation, approximation and applications. Commun. Nonlinear Sci. Numer. Simul. 241 (2015), 169-183.

18. K. V. Zhukovsky and H. M. SRivastava: Analytical solutions for heat diffusion beyond Fourier law. Applied Mathematics and Computation 293 (2017), 423-437.

19. L. Mahto, S. Abbas, M. Hafayed and H. M. Srivastava: Approximate Controllability of Sub-Diffusion Equation with Impulsive Conditio. Mathematics 7190 (2019), 1-16.

20. X. J. Yang, H. M. Srivastava, D. F. M. Torres and A. Debbouche: General Fractional-order Anomalous Diffusion with Non-singuler Power-Law Kernel. Thermal Science 211 (2017), 1-9.

21. I. Podlubny: Fractional Differential Equations. Academic Press, San Diego, 1999. 
Süleyman Çetinkaya

Faculty of Arts and Sciences

Department of Mathematics

41380 Kocaeli, Turkey

suleyman.cetinkaya@kocaeli.edu.tr

Ali Demir

Faculty of Arts and Sciences

Department of Mathematics

41380 Kocaeli, Turkey

ademir@kocaeli.edu.tr

Hülya Kodal Sevindir

Faculty of Arts and Sciences

Department of Mathematics

41380 Kocaeli, Turkey

hkodal@kocaeli.edu.tr 